\title{
Synthesis, characterization and antimicrobial studies of bio silica nanoparticles prepared from Cynodon dactylon L.: a green approach
}

\author{
REDDLA HARI BABU, PULICHERLA YUGANDHAR* and NATARU SAVITHRAMMA \\ Department of Botany, Sri Venkateswara University, Tirupati 517502, India \\ *Author for correspondence (yugandharbotany@gmail.com)
}

MS received 10 May 2017; accepted 21 September 2017; published online 16 May 2018

\begin{abstract}
The present study reports on a green approach method for synthesis of silica nanoparticles (SiNPs) from Cynodon dactylon. These SiNPs were characterized by using ultraviolet-visible (UV-Vis) spectrophotometer, Fouriertransform infrared (FT-IR), dynamic light scattering (DLS) and Zeta, X-ray diffractometer (XRD), scanning electron microscope (SEM), transmission electron microscopy (TEM) and evaluated its growth inhibitory efficacy against different microorganisms. These SiNPs showed a colour change pattern upon synthesis and indicated a broad peak at $350 \mathrm{~nm}$ when analysed by UV-Vis spectrophotometer. FT-IR analysis revealed the presence of Si content, and the appearance of phytochemicals such as primary amines of proteins, phenols were mainly responsible for capping and stabilization of SiNPs. DLS and Zeta potential studies revealed average size of $62.1 \mathrm{~nm}$ and $-23.3 \mathrm{mV}$ zeta potential value of nanoparticles. An XRD study showed a broad peak at $22^{\circ}$ of $2 \theta$ value and confirmed that the nanoparticles were amorphous in nature with $60 \mathrm{~nm}$ average size of particles. Higher magnification studies with SEM and TEM analysis revealed that the particles were poly-dispersed, spherical in shape and have the size range from 7 to $80 \mathrm{~nm}$ without any agglomeration among the particles. Energy dispersive X-ray analysis showed a 52.84 weight percentage of silica content in the sample, which indicates towering purity of the sample. The obtained nanoparticles were tested for growth inhibitory activity on different microbial pathogens, resulting in potential inhibitory activity. This study concluded that the plant $C$. dactylon was an excellent and reliable green source for production of potential bio antimicrobial SiNPs.
\end{abstract}

Keywords. Cynodon dactylon; green approach; silica nanoparticles; characterization; antimicrobial studies.

\section{Introduction}

Green synthesis of nanoparticles is an emerging field of nanotechnology, which attracts great attention in the fields of medicine, pharmaceutical, electrical, technology and other science research areas. Most of the nanoparticles are synthesized through different chemical and physical methods. These approaches are complicated, expensive and cause potential environmental and biological hazards [1]. Green synthesis of nanoparticles with the help of plants as reducing agents is an efficient, cost effective, fast and eco-friendly in manner [2]. In recent past, most of the scientists adopted green synthesis methods for the production of narrow-ranged particles, like calcium [3], copper [4], gold [5], iron [6], silica [7], silver [8] and zinc [9] from different medicinal plants. Among them silica nanoparticles (SiNPs) were recognized as important in the fields of chemistry, physics and biology due to their distinctive properties, which have different applications in the field of chemistry and physics. However, in terms of research undertaken and recognition of derivative uses of SiNPs were very scant in biology. While over some of the biological applications of SiNPs were evaluated at earlier times by efficient researchers in the fields such as, delivery of protein [10], delivery of gene [11], delivery of drug [12] and proved its biological activities, such as antifungal-antiviral [13], antioxidant [14], anticancer [15] and antibacterial [16] activities in the fields of biology and medicine. However, most of the above said activities in biology/medicine of SiNPs were proved by utilizing SiNPs synthesized from different chemical- and physicalmediated methods, which yields hazardous by-products to the environment.

Silica nanoparticles were mainly synthesized through different chemical and physical methods, such as sol-gel synthesis [17], chemical vapor condensation [18], flame synthesis [19], laser ablation [20] and reverse microemulsion [21]. These chemical and physical methods owe some disadvantages, like sol-gel synthesis has high raw materials cost, shrinkage and cracking may occur during the drying process and need very high temperature furnace or heating device [22]. The chemical vapor condensation method requires higher temperatures usually between 300 and $900^{\circ} \mathrm{C}$, slower growth rates and usage of precursors such as $\mathrm{Ni}(\mathrm{CO})_{4}$ is highly toxic, $\mathrm{B}_{2} \mathrm{H}_{6}$ and $\mathrm{SiCl}_{4}$ have an explosive and corrosive nature, respectively [23]. Difficulties in controlling particle size, morphology and phase composition are main 
disadvantages of flame synthesis [24]. The laser ablation method has limitations in wavelength of the laser impinging metallic target, duration of laser pulses, laser fluence, duration of ablation and effective liquid medium with or without the presence of surfactants [25]. Reverse microemulsion method limits its high cost and has difficulties in removal of surfactants from final products [17]. Apart from these methods, little research has been carried out on green synthesis method to synthesize SiNPs from plants and no research is undertaken so far from C. dactylon. Hence, the present work has been undertaken to synthesize SiNPs from $C$. dactylon, characterization of those SiNPs by using different analytical tools and test their antimicrobial activity against different microorganisms.

Cynodon dactylon (L.) Pers. is an annual grass herb that belongs to the family Poaceae and is locally known as Gerichagaddi in Telugu language. According to Indian mythology and medicinal scenario, the plant $C$. dactylon has important medicinal properties afterwards Ocimum sanctum. Not only that, Indian devotees considered this plant species as hallowed grass to adoration of Lord Ganesha from ancient times. Traditionally, the plant was used for the treatment of jaundice, skin infections [26], fevers and rheumatic pains [27]. It is a potent medicinal herb in Ayurveda, Homoeopathy and Unani systems of medicine to cure different ailments [28]. In the modern context, the plant has different biological activities that were proved by efficient scholars in terms of anti-hyperglycemic [29], anti-diabetic [30], hepatoprotective [31], antihyperlipedemic [32], anti-inflammatory [33], anthelmintic [34], antimicrobial [35], antiarthritic [27] and antioxidant activity [36].

\section{Materials and methods}

\subsection{Collection of plant material}

Healthy leaves of $C$. dactylon were collected from near and surrounding places of Sri Venkateswara University, Tirupati. The plant was authenticated by knowing its voucher specimen no: 196, deposited in Department of Botany, Sri Venkateswara University, Tirupati. The collected fresh leaves were washed 2-3 times with running tap water and shade dried up to 10 days under sterile conditions. These dried leaves were well-ground using kitchen blender and sieved with $5 \mathrm{~mm}$ sieve plate for synthesis of SiNPs.

\subsection{Chemicals}

Deionized Milli Q water (Merck Water Solutions, France), $\mathrm{HNO}_{3}$ and $\mathrm{NaOH}$ (Thermo Fisher Scientifics, India), nutrient broth agar medium, chemically synthesized SiNPs (Sigma Aldrich, Bangalore, India), gentamicin sterile discs (Himedia Chemicals, India) were mainly used in this study.

\subsection{Preparation of SiNPs}

Synthesis of SiNPs was followed by slight modifications of Adam et al [37] protocol. The well-ground $20 \mathrm{~g}$ of plant leaf powder was mixed with $500 \mathrm{ml}$ of $1 \mathrm{M} \mathrm{HNO}_{3}$ in a $1000-\mathrm{ml}$ Erlenmeyer conical flask and stirred it for $24 \mathrm{~h}$. This mixture was filtered and washed with distilled water to reach the $\mathrm{pH}$ up to 4.0-5.0. This solution was dried in an oven between 100 and $110^{\circ} \mathrm{C}$ for $12 \mathrm{~h}$. The evaporated turbid solution was stirred with $500 \mathrm{ml}$ of $1 \mathrm{M} \mathrm{NaOH}$ solution up to $24 \mathrm{~h}$ with a magnetic stirrer to reach the $\mathrm{pH}$ up to 12 . The obtained reaction mixture was separated with suction pump and titrated with $3 \mathrm{M} \mathrm{HNO}_{3}$ until the $\mathrm{pH}$ was attained up to 8.5-9.0. The contents were centrifuged at 5000 RPM for $10 \mathrm{~min}$ to separate biological admixtures. The contents were washed 3 to 4 times with distilled water and dried at $80^{\circ} \mathrm{C}$ in a hot air oven for $12 \mathrm{~h}$. The obtained powder was well-ground with a mortar and pestle, and was utilized for characterization and antimicrobial studies.

\subsection{Characterization of SiNPs}

Ultraviolet-visible (UV-Vis) spectroscopic characterization of obtained nanoparticles before drying was analysed by using UV-Vis Spectrophotometer 119, Systronics, India between the scan range of 300 to $700 \mathrm{~nm}$. Fourier-transform infrared (FT-IR) spectra was analysed by using an ALPHA interferometer (ECO-ATR), ZnSe liquid transmission cell of Bruker, Ettlingen, Karlsruhe, Germany, by $\mathrm{KBr}$ pellet method in the scan range of 4000 to $500 \mathrm{~cm}^{-1}$. Dynamic light scattering (DLS) and Zeta potential was carried out by using Nanopartica analyzer, Horiba SZ 100, Japan. X-ray diffraction (XRD) analysis was carried out with Siefert computerized $\mathrm{X}$-ray Diffractometer $3003 \mathrm{TT}$ by using $\mathrm{CuK}_{\alpha 1}$ radiation at $40 \mathrm{kV}$ and $20 \mathrm{~mA}$ at the $2 \theta$ range between $10^{\circ}$ and $80^{\circ}$, and determination of positions by using RAYFLEX-Analyze software. Scanning electron microscopic (SEM) analysis of nanoparticles along with elemental analysis was performed by using Hitachi S-4500 SEM attached with EDAX machine (Hitachi, Mexico) operating at $15 \mathrm{kV}$ passage of electric voltage. Transmission electron microscopic (TEM) analysis of nanoparticles was performed by using JEOL JEM-2010 (USA) with high-resolution transmission electron microscope operated between 80 and $200 \mathrm{kV}$ accelerating voltages.

\subsection{Antimicrobial studies}

The antimicrobial activity of synthesized nanoparticles was tested against four human pathogenic bacterial strains. Among them, two bacterial strains were Gram-positive such as, Bacillus subtilis ATCC 6633 and Staphylococcus aureus ATCC 6538. Two Gram-negative bacterial strains such as Escherichia coli ATCC 25922 and Pseudomonas aeruginosa ATCC 15442 were procured from the Department of Microbiology, Sri Venkateswara University, Tirupati. 
The disc diffusion assay method was followed for checking antimicrobial activity of synthesized SiNPs [38]. A quantity of $1 \mathrm{mg} \mathrm{ml}^{-1}$ of plant extract and $0.1 \%$ of DMSO were used as positive and negative controls, respectively. Gentamicin $\left(10 \mu \mathrm{g} \mathrm{ml}^{-1}\right.$ concentration) was used as a standard control. Further, to know the minimum inhibitory concentration of nanoparticles, serial concentrations of 5, 10, 20, 40 and $60 \mu \mathrm{g} \mathrm{ml}^{-1}$ concentrations of SiNPs were tested on all the selected microbes. Among them, 20 and $40 \mu \mathrm{g} \mathrm{ml}^{-1}$ of concentrations of SiNPs showed minimum inhibitory effect and $60 \mu \mathrm{g} \mathrm{ml}^{-1}$ concentration of SiNPs showed maximum inhibitory effect. Hence, in this study $60 \mu \mathrm{g} \mathrm{ml}^{-1}$ concentration of SiNPs was used to check the antimicrobial activity against different microbial organisms. To check the proximity of green synthesized SiNPs with chemically synthesized SiNPs, $60 \mu \mathrm{g} \mathrm{ml}^{-1}$ concentration of chemically synthesized SiNPs was used in this experiment. A quantity of $0.1 \%$ of DMSO was used to dissolve plant extract, green and chemically synthesized SiNPs. Sterile discs $(7 \mathrm{~mm})$ were prepared from Whatmann No. 1 filter paper and loaded with $20 \mu 1$ per disc of each aliquot of solutions under sterile conditions. Freshly prepared nutrient agar medium was poured into sterile Petri plates and later allowed for $30 \mathrm{~min}$ for solidification. The plates were swabbed with $100 \mu$ l of microbial cultures and placed the previously prepared discs on microbial-swabbed medium. This experiment was carried out in triplicates and were incubated at $37^{\circ} \mathrm{C}$ for $24-48 \mathrm{~h}$. The zone of inhibition was measured with the help of a scale and tabulated the results.

\section{Results and discussion}

The preliminary clue for confirmation of synthesized nanoparticles was SiNPs by its colour change pattern upon synthesis. Before the synthesis, the plant material was subjected to acid treatment by using $1 \mathrm{MHNO}_{3}$. This treatment removes impurities like cellulose, lignin materials and trace amount of metal ions in the form of liquid fraction (1). Finally, yields raw silicon dioxide $\left(\mathrm{SiO}_{2}\right)$ in the form of a pellet at the bottom of centrifuge tubes upon centrifugation. In our previous studies, $C$. dactylon leaf part showed good quantity of $\mathrm{SiO}_{2}$ in the form of phytoliths [39]. The liquid fraction was discarded and the pellet containing $\mathrm{SiO}_{2}$ was washed several times with distilled water. The content was stirred with $1 \mathrm{M} \mathrm{NaOH}$ to form sodium silicate and water molecules (2). The mixture was suction pumped and titrated with $3 \mathrm{M} \mathrm{HNO}_{3}$ to get the pure form of $\mathrm{SiO}_{2}$, sodium nitrate and water molecules (3). When $3 \mathrm{M}$ $\mathrm{HNO}_{3}$ solution was added dropwise to the suction pumped plant mixture, the colour change pattern of the mixture was observed gradually from brown to whitish precipitate.

$$
\begin{aligned}
& \text { Plant material }+\mathrm{HNO}_{3} \longrightarrow \text { liquid fraction }+\mathrm{SiO}_{2} \\
& 2 \mathrm{NaOH}+\mathrm{SiO}_{2} \longrightarrow \mathrm{Na}_{2} \mathrm{SiO}_{3}+\mathrm{H}_{2} \mathrm{O} \\
& \mathrm{Na}_{2} \mathrm{SiO}_{3}+2 \mathrm{HNO}_{3} \longrightarrow \mathrm{SiO}_{2}+2 \mathrm{NaNO}_{3}+\mathrm{H}_{2} \mathrm{O}
\end{aligned}
$$

These solutions were analysed with the help of UV-Vis spectrophotometer, which displayed a broad peak at $350 \mathrm{~nm}$ due to the surface plasmon resonance nature of nanoparticles in the reaction medium. These nanoparticles absorb light at different wavelengths and are excited to give a broad peak (figure 1a). Similar type of results were observed in SiNPs synthesized from rice husk ash by thermolysis method [40]. FT-IR spectroscopic analysis of SiNPs showed four peaks: at $1076 \mathrm{~cm}^{-1}$ corresponds to $\mathrm{C}-\mathrm{N}$ stretch of aliphatic amines, $949 \mathrm{~cm}^{-1}$ corresponds to $\mathrm{O}-\mathrm{H}$ bend of carboxylic acids, $798 \mathrm{~cm}^{-1}$ corresponds to $\mathrm{Si}-\mathrm{O}$ bending vibration, here the oxygen atom moves at right angles to $\mathrm{Si}-\mathrm{Si}$ lines to form $\mathrm{Si}-\mathrm{O}-\mathrm{Si}$ plane and $511 \mathrm{~cm}^{-1}$ corresponds to $\mathrm{Si}-\mathrm{O}$ rocking vibration, here the oxygen atom moves perpendicular to the Si-O-Si plane (figure 1b). These kind of data were matched with SiNPs derived from rice husk [41]. This FT-IR spectroscopic study revealed that the obtained $\mathrm{Si}-$ $\mathrm{O}-\mathrm{Si}$ plane of silica material was converted into spherical shaped SiNPs by encapsulating $\mathrm{C}-\mathrm{N}$ stretch of amines, a subgroup of proteins and $\mathrm{O}-\mathrm{H}$ bend of carboxylic acids, a subgroup of phenols. These phytochemicals stabilize and evade agglomeration between the nanoparticles. DLS analysis of synthesized SiNPs revealed $62.1 \mathrm{~nm}$ average size and $-23.3 \mathrm{mV}$ of zeta potential value. It indicates the higher stability of nanoparticles (figure 1c and d). This DLS and Zeta potential study of nanoparticles determines particle size and its stability by measuring rapid changes of scattered light in a solution or suspended media. It denotes the opted method as a best suitable method for green synthesis of SiNPs.

Crystallographic studies of synthesized nanoparticles with XRD instrument displayed a broad and high intensity peak at $22^{\circ}$ of $2 \theta$ values of $x$-axis corresponding to the amorphous nature of SiNPs (figure $2 \mathrm{a}$ ). This result was correlated with JCPDS file No. 01-0787 of the Joint Committee on Powder Diffraction Standards. The obtained XRD peak was broad due to small size and incomplete inner structure of particles. Absence of other sharp peaks proves the amorphous nature of synthesized SiNPs. The same type of results were observed in a rice husk-mediated synthesis of SiNPs [37]. The $60 \mathrm{~nm}$ average size of the particles was calculated by producing fullwidth half-maximum (FWHM) value taken at $22^{\circ}$ of $x$-axis by using the Debye-Scherrer equation $(D=k \lambda / \beta \cos \theta)$. The $5 \mu \mathrm{m}$ resolution studies of SEM analysis revealed polydispersed, spherical shaped particles with a size range from 60 to $80 \mathrm{~nm}$ and most of the particles were settled in nonagglomerated state (figure 2b). Energy Dispersive X-Ray analysis showed a 52.84 weight percentage of silica along with $47.16 \%$ of oxygen (figure $2 \mathrm{c}$ ). More than $50 \%$ of silica content found in the sample indicates high purity of SiNPs produced through this process. These EDAX data are matched with FT-IR studies to represent only silica and oxygen contents deposited in the form of Si-O-Si. The nanoparticle size analysis study of SEM micrographs revealed $67 \mathrm{~nm}$ average size of the particles with 0.99 adjacent ' $R{ }^{2}$ ' value (figure $2 \mathrm{~d}$ ). The selected area electron dispersion (SAED) pattern of 

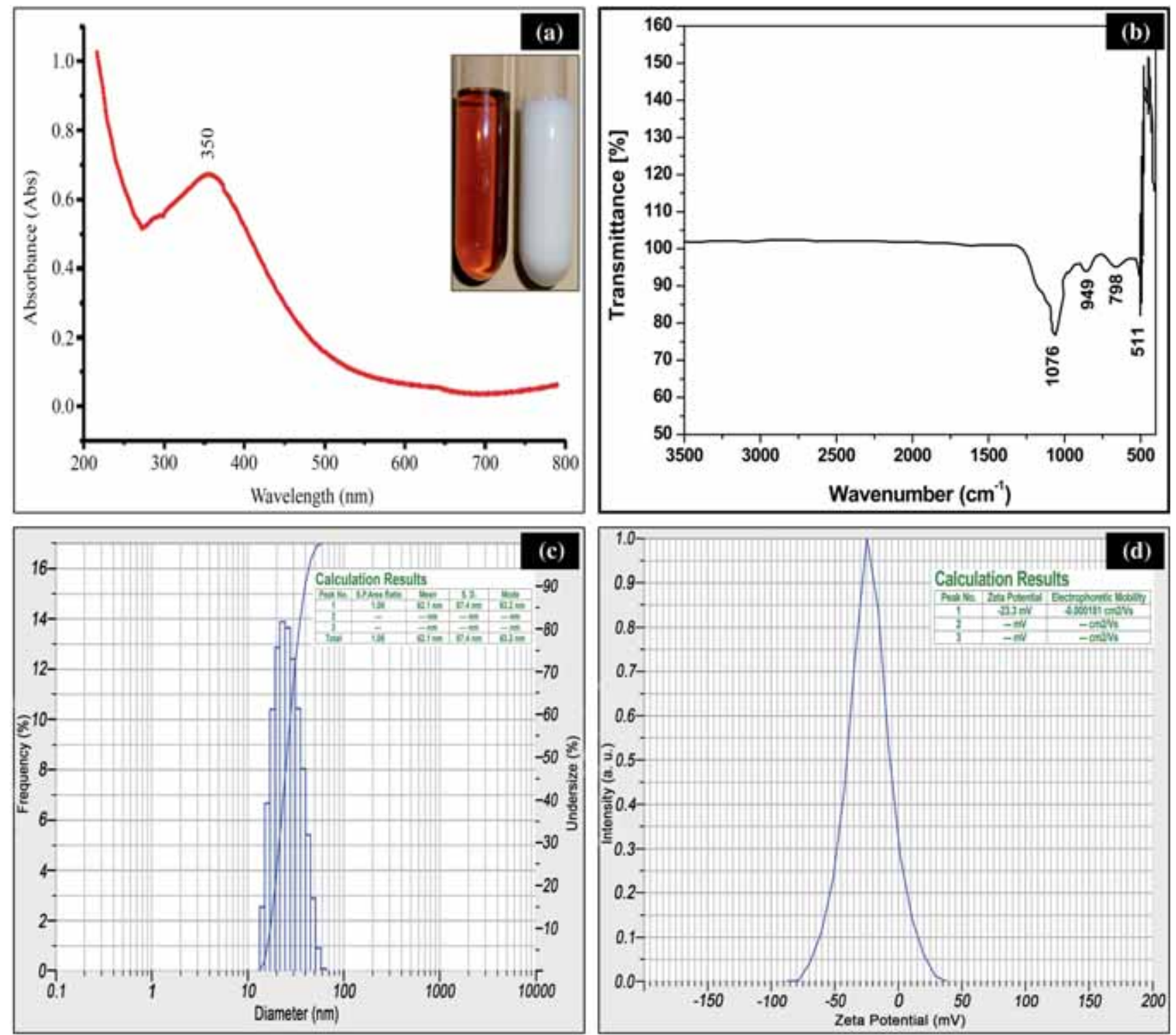

Figure 1. (a) UV-Vis spectroscopy analysis of synthesized SiNPs represents a broad peak at $350 \mathrm{~nm}$. Inset photograph represents colour change pattern. (b) FT-IR spectroscopy analysis confirms SiNPs synthesized by the involvement of primary amines and phenols. (c) DLS analysis reveals $62.1 \mathrm{~nm}$ average size. (d) Zeta potential analysis reveals $-23.3 \mathrm{mV}$ of negative value.

transmission electron microscopy (TEM) analysis showed a fringe array of spots, which correspond to the results of XRD $2 \theta$ value of $22^{\circ}$, and Bragg reflections revealed the amorphous nature of particles (figure $3 \mathrm{a}$ ). The 20 and $50 \mathrm{~nm}$ higher magnification studies by TEM analysis revealed that the particles were poly-dispersed in condition, spherical in shape and have the size range from 7 to $12 \mathrm{~nm}$ (figure $3 \mathrm{~b}$ and c). The nanoparticle size analysis study of TEM micrographs revealed $4 \mathrm{~nm}$ average size of the particles with 0.97 adjacent ' $R$ ' ' value calculated by using the Gauss equation (figure $3 \mathrm{~d}$ ).

Antimicrobial studies of green synthesized SiNPs showed potential antimicrobial activity against different microbial pathogens. When the green synthesized SiNPs with plant extract was compared with chemically synthesized SiNPs, the green synthesized SiNPs displayed better results. In chemicalor physical-mediated protocols, difficulties with operational methods and effects of perilous chemicals, the green approach method is reliable for synthesis of SiNPs in a cost-effective and eco-friendly manner. Not only that, these nanoparticles showed reliable antimicrobial activity when compared with chemically synthesized SiNPs. This study concludes that the green synthesized method implied better results than the chemically mediated method. The highest growth inhibitory activity of bio nanoparticles was observed on $E$. coli followed by $P$. aeruginosa, B. subtilis and $S$. aureus 

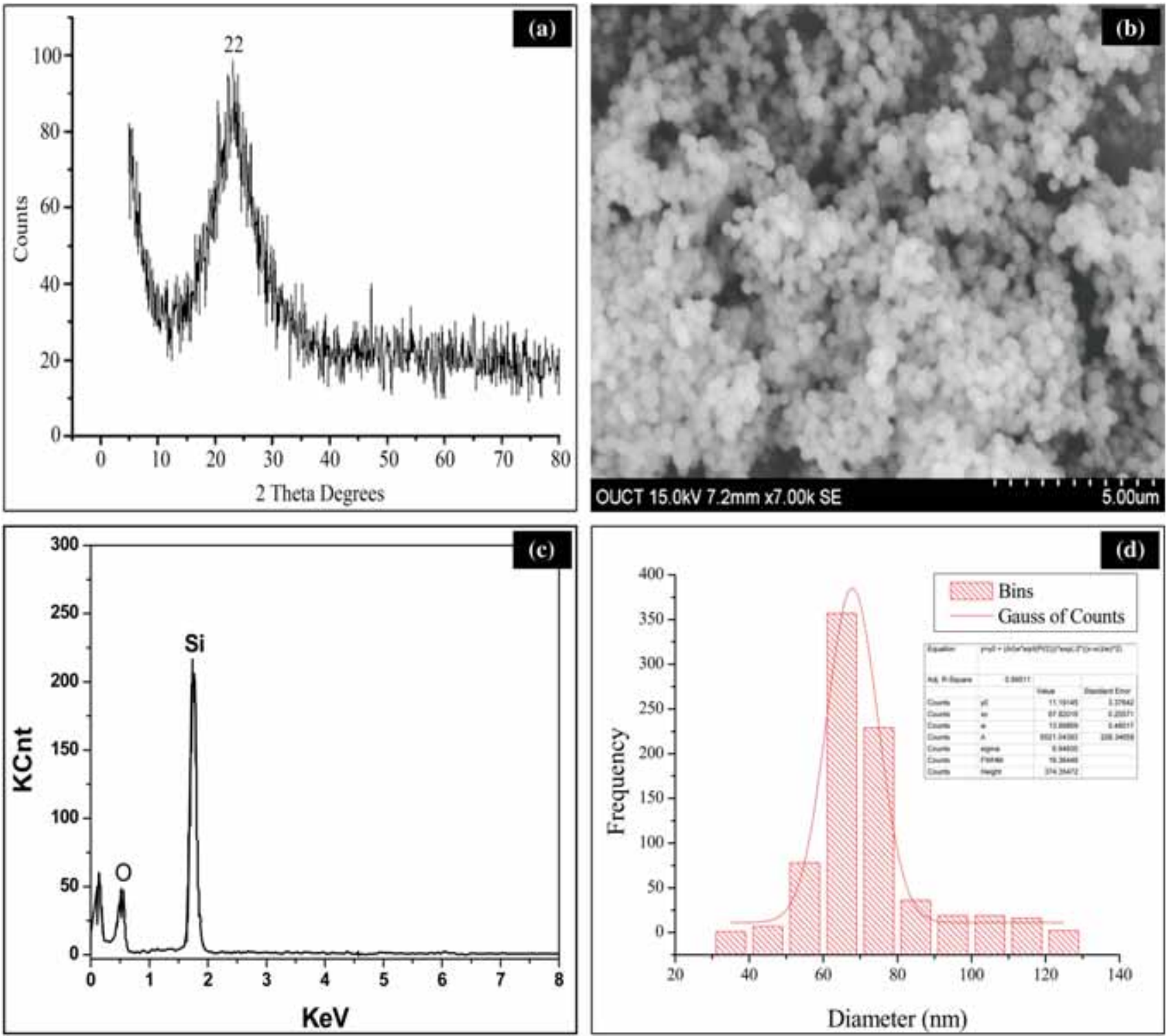

Figure 2. (a) XRD analysis of SiNPs shows highest intensive peak at $22^{\circ}$ and confirms amorphous nature of particles. (b) SEM analysis displays poly-dispersed, spherical shaped, 60-80 nm sized, non-agglomerated SiNPs. (c) Energy dispersive X-ray analysis reveals 52.84 weight percentage of silica content. (d) Particle size analysis reveals $67 \mathrm{~nm}$ average size.

(figure 4; table 1). This type of results were observed in gold-doped SiNPs [16] as well as the polymer-grafted SiNPs synthesized through chemically mediated protocols [42]. The antimicrobial effect of green synthesized SiNPs attributed higher activity on Gram-negative bacterial stains, such as $E$. coli and $P$. aeruginosa. Whereas the antimicrobial activity was comparatively less in the case of Gram-positive bacterial strains such as $B$. subtilis and $S$. aureus. The Gram-positive bacteria allowed less SiNPs to reach the cytoplasmic membrane than Gram-negative bacteria. It may be due to the Gram-positive bacteria that have more layers of peptidoglycon in the cell walls of bacteria, which is a complex structure of bacteria, often contains teichoic acids or lipoteichoic acids that modify the cell walls of bacteria into a rigid obstacle for the penetration of nanoparticles. These Gram-positive bacterial strains have strong negative conditions for penetration of SiNPs on multiple layers of peptidoglycon when compared to the cell walls of Gram-negative bacteria. This is the main reason for obtaining greater antimicrobial activity in Gram-negative bacteria when compared with Gram-positive bacteria. The same type of results were observed in SiNPs prepared from Adansonia digitata pulp extract [43].

This study revealed that opted green synthesis method is the easiest way for synthesis of SiNPs by an eco-friendly and cost-effective approach by utilizing green leaves of C. dactylon. Many researchers succeeded for synthesis of these nanoparticles by using silicon tetrachloride $\left(\mathrm{SiCl}_{4}\right)$, 

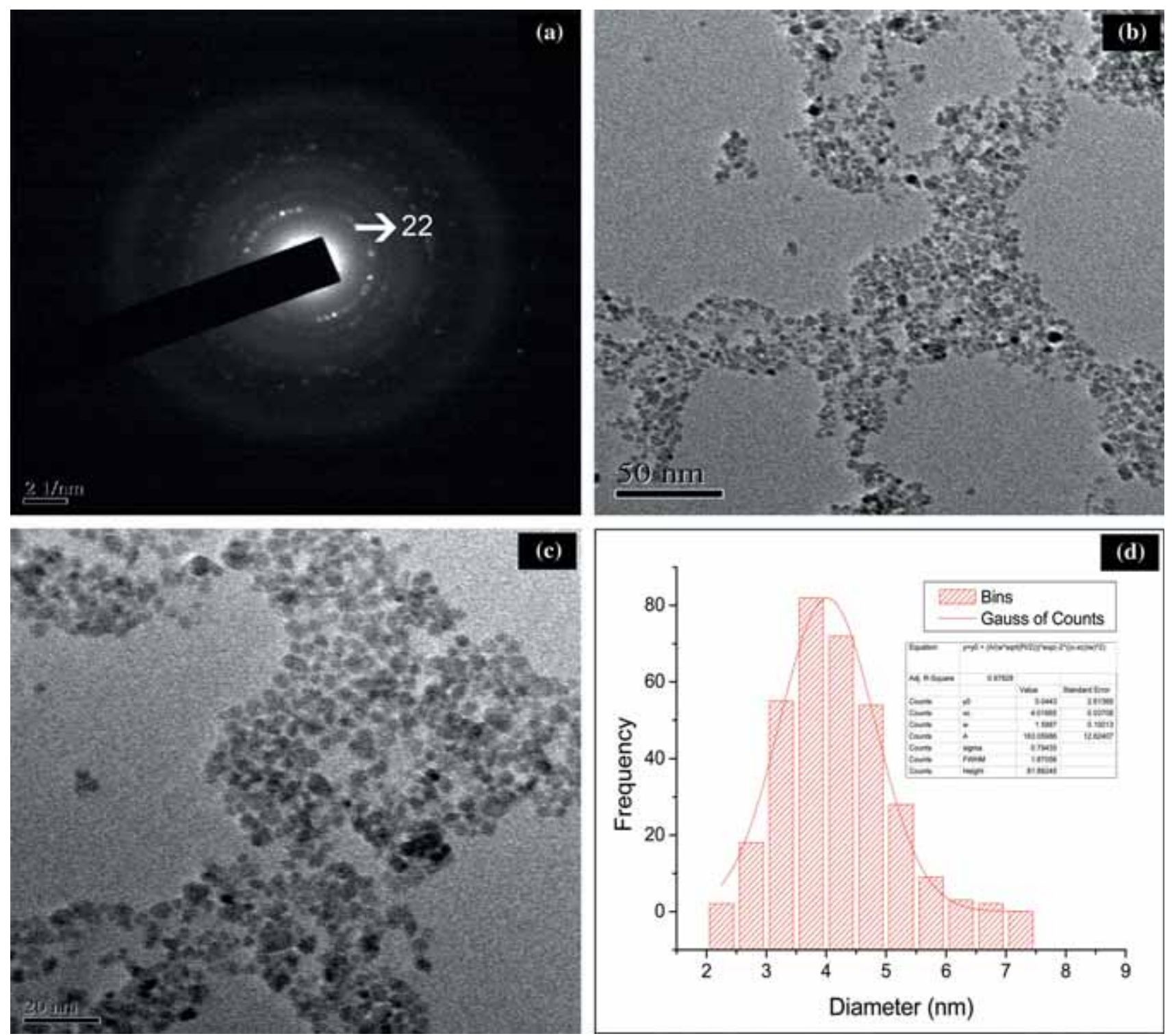

Figure 3. (a) SAED pattern of nanoparticles shows fringe array of spots. (b) $50 \mathrm{~nm}$ magnification studies reveal spherical shape of particles with $7-12 \mathrm{~nm}$ size. (c) $20 \mathrm{~nm}$ magnification studies show non-agglomerated particles. (d) Particle size analysis reveals $4 \mathrm{~nm}$ average size.

tetraethyl orthosilicate $\left(\mathrm{SiC}_{8} \mathrm{H}_{20} \mathrm{O}_{4}\right)$ and rice husks as silica source. However, the utilization of chemicals such as $\mathrm{SiCl}_{4}$ and $\mathrm{SiC}_{8} \mathrm{H}_{20} \mathrm{O}_{4}$ causes severe health hazards. $\mathrm{SiCl}_{4}$ is a clear corrosive chemical, colourless, fuming liquid with an irritating odor. In 1984, spilling of $\mathrm{SiCl}_{4}$ in industrial park noticed that the contact of chemical irritates and burns the skin and eye. Inhalation of $\mathrm{SiCl}_{4}$ fumes irritates nose, throat and lungs. Higher exposures may cause pulmonary edema in the lungs and may affect nervous system [44]. $\mathrm{SiC}_{8} \mathrm{H}_{20} \mathrm{O}_{4}$ is a colourless liquid and causes eye irritation when exposed, leading to corneal damage after $24 \mathrm{~h}$ and may lead to conjunctivitis after repeated exposures. The contact of chemical with skin may cause redness, swelling and blistering. Inhalation through nostrils causes respiratory irritation and higher exposures may cause narcosis, liver, kidney damage and leads to anemia [45]. Utilization of rice husks as the silica source was better than the other sources, because the plant accumulates silica contents in their tissues to pertain rigid nature and was prone from sudden environmental damages. These depositions were utilized as a silica source for synthesis of SiNPs. With inspiration from rice husk, the present study also succeeded to produce SiNPs by using C. dactylon as silica source, which is a good source of silica content stored in its foliar parts. The plants, rice and $C$. dactylon are from the same family and accumulate silica contents in the form of phytoliths in leaves, husks and stem parts of the plant. In this practice, we succeed to produce SiNPs from $C$. dactylon and it paves a way to further step towards the synthesis of SiNPs in a green approach method from different biological sources. 

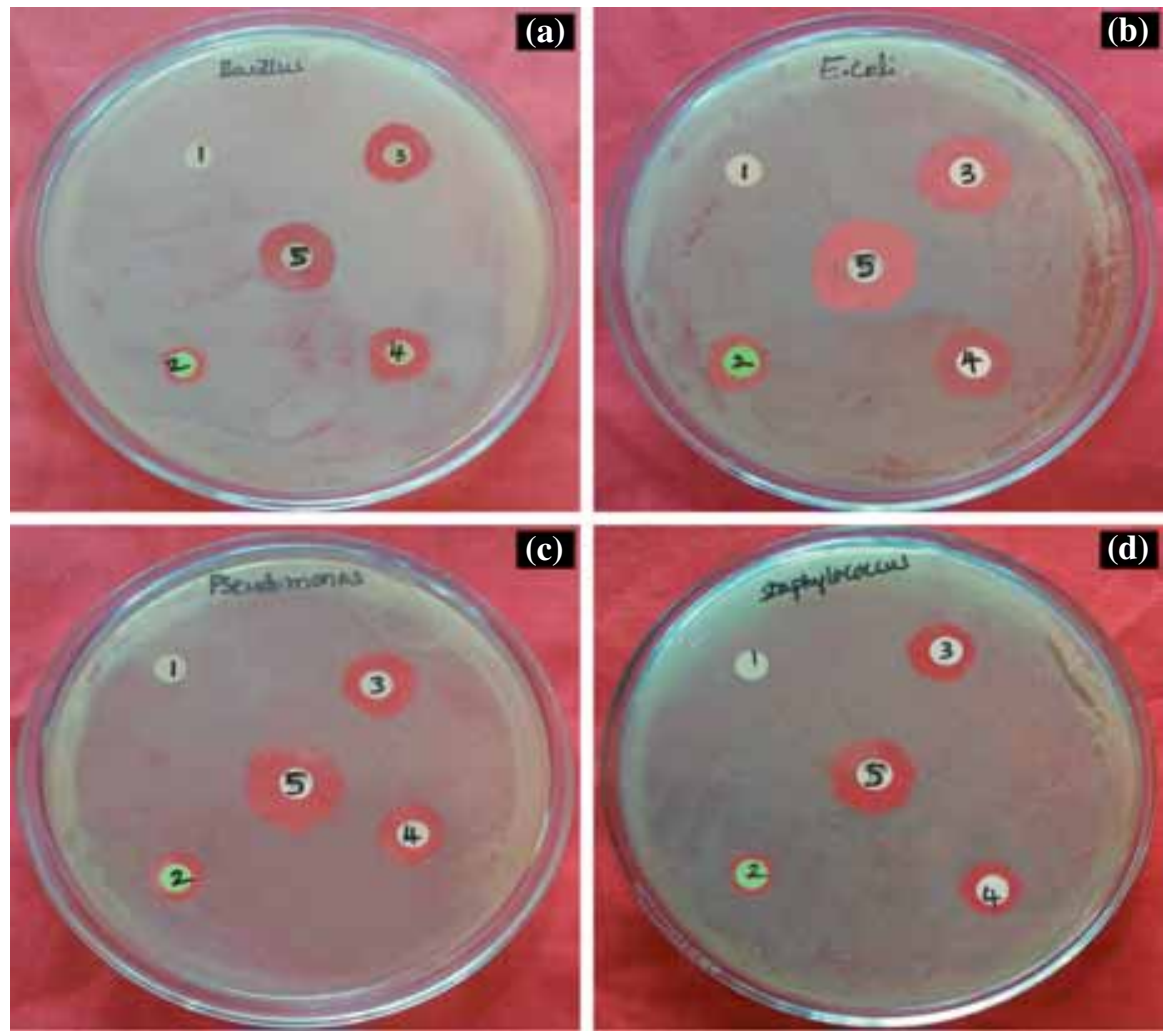

Figure 4. Antimicrobial activity of green synthesized SiNPs. (a) B. subtilis, (b) E. coli, (c) P. aeruginosa, (d) S. aureus. 1: DMSO, 2: plant extract, 3: green synthesized SiNPs, 4: chemically synthesized SiNPs and 5: gentamicin.

Table 1. Antimicrobial activity of green synthesized silica nanoparticles from C. dactylon.

\begin{tabular}{lcrcrr}
\hline Name of the organism & DMSO & Plant extract & Green synthesized SiNPs & Chemical synthesized SiNPs & Gentamicin \\
\hline B. subtilis & 0.0 & $8.1 \pm 0.17$ & $10.4 \pm 0.31$ & $9.9 \pm 0.14$ & $12.7 \pm 0.24$ \\
E. coli & 0.0 & $10.2 \pm 0.28$ & $14.2 \pm 0.35$ & $12.1 \pm 0.28$ & $15.2 \pm 0.28$ \\
P. aeruginosa & 0.0 & $9.1 \pm 0.24$ & $13.1 \pm 0.24$ & $11.6 \pm 0.16$ & $15.0 \pm 0.17$ \\
S. aureus & 0.0 & $8.0 \pm 0.10$ & $10.3 \pm 0.07$ & $9.6 \pm 0.17$ & $12.5 \pm 0.21$ \\
\hline
\end{tabular}

\section{Conclusion}

In this study an attempt has been made to synthesize SiNPs from the leaves of $C$. dactylon, which act as environmentally benign silica source for synthesis of SiNPs without involvement of any toxic chemicals or corrosive gasses. The constraints aroused with different chemical/physical methods, the present green approach method is reliable for synthesis of SiNPs. The synthesized SiNPs from $C$. dactylon were spherical in shape, poly-dispersed in nature, having the size range from 7 to $80 \mathrm{~nm}$ without any agglomeration between the particles. This was proved by using different analytical and microscopic techniques. In this study, some of the results were correlated vice versa, such as FT-IR studies displayed silica content in the form of $\mathrm{Si}-\mathrm{O}-\mathrm{Si}$ plane and EDAX studies quantify 52.84 weight percentage of silica content. Fringe array of spots appeared in SAED pattern was coincided with a broad peak obtained in XRD studies, which revealed that the particles were amorphous in nature. The present opted method is chemically free while over silica precursors like silicon tetrachloride or tetraethyl orthosilicate and devoid of the use of calcination procedure. The plant itself acts as silica source, which stores silica in the form of phytoliths in epidermal parts of the plant. Based on these studies, we conclude that the plant $C$. dactylon acts as an excellent green source for the production of narrow range of SiNPs with synergistic growth inhibitory activities on different microbial pathogens. All the characterization results in this green approach method are matched with different chemical/physical-mediated methods without the utilization of harmful chemicals or corrosive 
gasses. This study makes out the best way to understand green approach method to synthesize SiNPs and its antimicrobial activity. Not only that, it paves a futuristic way for synthesizing SiNPs from plants that may store silica material in the form of phytoliths.

\section{Acknowledgements}

We are highly grateful to UGC-BSR for providing RFSMS fellowships and highly thankful to Prof. C Suresh Reddy, Department of Chemistry, S.V. University for providing FT-IR analysis and JNTU, Hyderabad, for providing characterization facilities. Finally, greatly acknowledged to Prof. K Suma Kiran, Department of English, S.V. University, for assisting in language corrections.

\section{References}

[1] Yugandhar P, Haribabu R and Savithramma N 2015 Biotech. 5 1031

[2] Yugandhar P and Savithramma N 2015 Nano Biomed. Eng. 7 29

[3] Yugandhar P and Savithramma N 2013 Int. J. Adv. Res. 189

[4] Shende S, Ingle A P, Gade A and Rai M 2015 World J. Microbiol. Biotechnol. 31865

[5] Gopinath K, Gowri S, Karthika V and Arumugam A 2014 J. Nanostruct. Chem. 41

[6] Naseem T and Farrukh M A 2015 J. Chem. 20151

[7] Athinarayanan J, Periasamy V S, Alhazmi M, Alatiah K A and Alshatwi A A 2015 Ceram. Int. 41275

[8] Yugandhar P and Savithramma N 2016 Appl. Nanosci. 6 223

[9] Bala N, Saha S, Chakraborty M, Maiti M, Das S, Basu R et al 2015 RSC Adv. 54993

[10] Fang I J and Trewyn B G 2012 Methods Enzymol. 508 41

[11] Hartono S B, Phuoc N T, Yu M, Jia Z, Monteiro M J, Qiao S et al 2014 J. Mater. Chem. B 2718

[12] Koskimaki J, Tarkia M, Ahtola-Satila T, Saloranta L, Simola O, Forsback A P et al 2015 Biomed. Res. Int. 2015715

[13] Botequim D, Maia J, Lino M M, Lopes L M, Simoes P N, Ilharco L M et al 2012 Langmuir 287646

[14] Shaji J and Varkey D 2013 J. Pharm. Invest. 43405

[15] Jambhrunkar S, Qu Z, Popat A, Yang J, Noonan O, Acauan L et al 2014 Mol. Pharm. 113642

[16] Shanmugan S, Ramalingam R J and Mutharasu D 2015 Synth. React. Inorg. Met. Org. Nano-Met. Chem. 45304

[17] Rahman I A and Padavettan V 2012 J. Nanomater. 20121

[18] Yu J H, Lee C W, Im S S and Lee J 2003 Rev. Adv. Mater. Sci. 455
[19] Yue R, Meng D, Ni Y, Jia Y, Liu G, Yang J et al 2013 Powder Technol. 235909

[20] Sana N O, Kursungoz C, Tumtas Y, Yasa O, Ortac B and Tekinay T 2014 Particuology 1729

[21] Lin C H, Chang J H, Yeh Y Q, Wu S H, Liu Y H and Mou C Y 2015 Nanoscale 79614

[22] Carter C B and Norton M G 2007Sols, gels, and organic chemistry (New York: Springer)

[23] Creighton J R and Ho P 2001 Introduction to chemical vapor deposition (CVD) (Materials Park, Ohio: ASM International)

[24] Klabunde K J 2001 Nanoscale materials in chemistry (New York: Wiley)

[25] Iravani S, Korbekandi H, Mirmohammadi S V and Zolfaghari B 2014 Res. Pharm. Sci. 9385

[26] Bharati D, Sonawane S A, Amrutkar M P, Undale V R, Wankhade A M and Bhosale A V 2009 Pharmacologyonline 21

[27] Bhangale J and Acharya S 2014 Indian J. Exp. Biol. 52215

[28] Nagori B P and Solanki R 2011 Res. J. Med. Plant 5508

[29] Mahesh N and Brahatheeswaran D 2007 Asian J. Biochem. 2 66

[30] Jarald E E, Joshi S B and Jain D C 2008 Indian J. Exp. Biol. 46660

[31] Prabha D C and Annapoorani S 2009 J. Bio. Sci. 1727

[32] Kaup S R, Arunkumar N, Bernhardt L K, Vasavi R G, Shetty S S, Pai S R et al 2011 Genomic Med. Biomarkers Health Sci. 398

[33] Garg V K and Paliwal S K 2011 Int. J. Pharmacol. 7370

[34] Abhishek B and Anita T 2012 J. Pharmacogn. Phytochem. 11

[35] Abdullah S, Gobilik J and Chong K P 2013 In vitro antimicrobial activity of Cynodon dactylon (L.) Pers. (bermuda) against selected pathogens (Boston: Springer)

[36] Koushik O S, Srinivasa Babu P and Karthikeyan R 2015 Transl. Biomed. 63

[37] Adam F, Chew T S and Andas J 2011 J. Sol-Gel Sci. Technol. $\mathbf{5 9} 580$

[38] Cruickshank R 1986 Medical microbiology: a guide to diagnosis and control of infection (Livingston: Edinburgh)

[39] Babu R H, Yugandhar P and Savithramma N 2015 Int. J. Plant Anim. Environ. Sci. 56

[40] Djangang C N, Mlowe S, Njopwouo D and Revaprasadu N 2015 J. Appl. Chem. 41218

[41] Alshatwi A A, Athinarayanan J and Periasamy V S 2015 Mater. Sci. Eng. C Mater. Biol. Appl. 478

[42] Kawahara T, Takeuchi Y, Wei G, Shirai K, Yamauchi T and Tsubokawa N 2009 Polymer J. 41744

[43] Kumar C M K, Yugandhar P and Savithramma N 2016 J. Intercult. Ethnopharmacol. 579

[44] Kizer K W, Garb L G and Hine C H 1984 J. Occup. Med. 26 33

[45] Material safety data sheet (last accessed date: 22.04.2010) http://datasheets.scbt.com/sds/wpna/en/sc-253664.pdf 